\title{
G2 Phase Process
}

National Cancer Institute

\section{Source}

National Cancer Institute. G2 Phase Process. NCI Thesaurus. Code C17356.

Phase of the cell cycle following DNA synthesis and preceding mitosis (Gap 2 phase). The chromosomes are tetraploid in this phase. 